COMMUNICATIONS IN

ANALYSIS AND GEOMETRY

Volume 14, Number 1, 135-161, 2006

\title{
Stability conditions and the braid group
}

\author{
R. P. Thomas
}

We find stability conditions $[6,3]$ on some derived categories of differential graded modules over a graded algebra studied in $[12,10]$. This category arises in both derived Fukaya categories and derived categories of coherent sheaves. This gives the first examples of stability conditions on the A-model side of mirror symmetry, where the triangulated category is not naturally the derived category of an abelian category. The existence of stability conditions, however, gives many such abelian categories, as predicted by mirror symmetry.

In our examples in 2 dimensions, we completely describe a connected component of the space of stability conditions. It is the universal cover of the configuration space $C_{k+1}^{0}$ of $k+1$ points in $\mathbb{C}$ with centre of mass zero, with deck transformations the braid group action of $[10,15]$. This gives a geometric origin for these braid group actions and their faithfulness, and axiomatises the proposal for stability of Lagrangians in [18] and the example proved by mean curvature flow in [19].

\section{Introduction.}

This paper presents a result in pure algebra, but one which is motivated entirely by geometry and physics, especially mirror symmetry. It gives examples of Douglas and Bridgeland's notion of stability conditions for triangulated categories $[5,6,1,3]$, and draws together and axiomatises many of the known tests of Kontsevich's homological mirror conjecture [11] (for instance on stability of Lagrangians in Fukaya categories [18], Shapere-Vafa's examples of special Lagrangians [16, 19], and braid groups of monodromies $[10,15])$. We explain some of the geometry and physics background, distill this into a purely algebraic setup, and then apply the axioms of stability conditions [3] to this problem. The result, which can be read independently of the previous sections, is a description of (a connected component of) the space of stability conditions on a certain natural triangulated category arising in many areas of geometry and algebra $[12,10]$, giving both an axiomatic justification for the conjectures and results of $[18,19]$, and a geometric "explanation" or origin for the faithful braid group actions of $[10,15]$, at least 
in dimension 2. There are other braid group actions in [17]; it would be nice to see them arise from stability conditions also.

\section{Geometry and physics background.}

Consider the following much-studied $[10,16,19]$ affine algebraic variety $X=$ $X^{N}(p)$ :

$$
\left\{\sum_{i=1}^{N} x_{i}^{2}=p(t)\right\} \subseteq \mathbb{C}^{N} \times \mathbb{C},
$$

where $p$ is some degree $k+1$ polynomial in $t \in \mathbb{C}$ with only simple zeros. There is a natural Kähler form $\omega$ restricted from $\mathbb{C}^{N+1}$, and a nowhere-zero holomorphic volume form $\Omega_{p}$ ("almost Calabi-Yau" structure) given by taking the Poincaré residue $[7, \mathrm{p}$. 147] of the meromorphic form $d x_{1} \ldots d x_{N} d t /\left(\sum x_{i}^{2}-p(t)\right)$ on $\mathbb{C}^{N+1}$; this can be written as

$$
\Omega=\Omega_{p}=(-1)^{N+i+1} \frac{\left.d x_{1} \ldots \widehat{d x}_{i} \ldots d x_{N} d t\right|_{X^{N}}}{2 x_{i}}=\frac{\left.d x_{1} \ldots d x_{N}\right|_{X^{N}}}{\dot{p}(t)}
$$

for any $i$ (so where $x_{i}=0 \forall i$, we can use the second expression). Here, $\widehat{d x}_{i}$ means that we omit the $d x_{i}$ term from the wedge product.

Each smooth fibre over $t \in \mathbb{C}$ is an affine quadric with a natural Lagrangian $S^{N-1}$ "real" slice, namely the intersection of the fibre with the slice

$$
x_{i} \in \sqrt{p(t)} \mathbb{R} \quad \forall i .
$$

It is invariant under the obvious $O(N)$ action on $X^{N}$, and is the vanishing cycle of every singular fibre (i.e. the fibres over the roots of $p$ ). Therefore, any path $\gamma:[0,1] \rightarrow \mathbb{C}$ from one zero of $p$ to another (avoiding zeros of $p$ in its interior) lifts to give a canonical $O(N)$-invariant Lagrangian $N$-sphere, $S^{N-1}$-fibred over $\gamma$ except at the endpoints where it closes up.

Picking an ordering of the $k+1$ zeros of $p$, we can pick $k$ consecutive paths in $\mathbb{C}$ joining one zero to the next, forming a so-called " $A_{k}$-chain" of paths. The corresponding Lagrangian $N$-spheres $\left\{L_{i}\right\}_{i=1}^{k}$ in $X^{N}$ (the vanishing cycles of the $A_{k}$-singularity; the singularity obtained by putting $p(t)=t^{k+1}$ in (2.1)) form a basis for its homology and define an $A_{k}$-chain of Lagrangians [13]. That is, their geometric intersections satisfy

$$
\left|L_{i} . L_{j}\right|=1, \quad|i-j|=1, \quad\left|L_{i} . L_{j}\right|=0, \quad|i-j|>1 .
$$


Choosing their gradings [14] appropriately, we can arrange that their Floer cohomologies satisfy

$$
\begin{aligned}
H F^{0}\left(L_{i}, L_{i}\right) & =\mathbb{C}=H F^{N}\left(L_{i}, L_{i}\right), \\
H F^{1}\left(L_{i}, L_{i+1}\right) & =\mathbb{C}=H F^{N-1}\left(L_{i+1}, L_{i}\right),
\end{aligned}
$$

for all $i$, with all other groups zero.

As $p$ varies, all of these varieties $X_{p}$ are isomorphic as symplectic manifolds, even though as complex manifolds they are varying. Thus, around loops in the space of polynomials with simple zeros $\{p\}$, we get monodromy in the symplectic automorphism group $\operatorname{Aut}(X, \omega)$. Scaling $p$ makes no difference to the symplectic geometry, nor, in dimension $N=2$ to the graded symplectic geometry, as $\Omega_{p}(2.2)$ is left unaltered. Similarly, everything is invariant under translations in the $t$-plane; dividing by these reparametrisations leaves us with the (simple) roots of $p$ normalised (say) to have centre of mass zero. That is, we get the configuration space $C_{k+1}^{0}$ of $k+1$ points of mean zero in $\mathbb{C}$, with fundamental group the braid group $B_{k}$. This gives a representation of $B_{k} \rightarrow \operatorname{Aut}(X, \omega)$, generated by "generalised Dehn twists" about the vanishing cycles; this amounts to "twisting" about the Lagrangian fibred over a path between two roots of $p$ by rotating the two points in $\mathbb{C}$ about each other; i.e. it arises from the usual description of $B_{k}$ lifted from $\mathbb{C}$ (with $k+1$ marked points) to $X$. In fact, these Dehn twists give such a braid group action on any symplectic manifold with an $A_{k}$-chain of Lagrangian spheres [13], and so this a local model for symplectic automorphisms of compact symplectic manifolds, in particular Calabi-Yau manifolds. This induces a braid group action on the derived Fukaya category $D^{b}(F u k(X))$ of $X$ (though one must check first, as in [14], that the symplectomorphisms lift naturally to the graded symplectomorphism group).

Under mirror symmetry (and there is a proposal for the mirror of this $A_{k}$-smoothing in [15] Section 3f), we cannot expect a braid group action of holomorphic automorphisms of any mirror complex manifold $Y$; there are in general very few holomorphic automorphisms of Calabi-Yau manifolds, and it is one of Kontsevich's great insights that the mirror is really the bounded derived category $D^{b}(Y) \cong D^{b}(F u k(X))$ of coherent sheaves on the CalabiYau rather than the Calabi-Yau itself; in this way, the automorphisms can be matched, so things work in families.

So if the only vestiges of our geometric picture above that remain under the mirror map are categorical, we would like to see the varying complex structure (given by the polynomial $p$ ) that induces the braid monodromy at the level of the (purely symplectically defined) derived Fukaya category. 
How one "ought" to do this has long been known to physicists, and was described to me many years ago by Eric Zaslow. Namely, one should follow the D-branes in $D^{b}(F u k(Y))$; these depend on the complex structure, and are thought to be the special Lagrangians (Lagrangians $L$ for which the $N$-form $\left.(2.2) \Omega\right|_{L}$ has constant phase on $L$ ). On going round a loop in complex structure moduli space, the set of special Lagrangians undergoes monodromy which one might hope extends uniquely to the full derived category. Similarly, in the mirror picture, the D-branes in $D^{b}(X)$ depend on the Kähler structure, and are to a first approximation the stable bundles that satisfy an appropriately perturbed Hermitian-Yang-Mills equation. In [18], this picture was mirrored to give an appropriate notion of stability for Lagrangians which should conjecturally be equivalent to the Lagrangian being a Hamiltonian deformation of a (unique) special Lagrangian. In the case of the Lagrangians of our above example, this can all be illustrated very simply [19], at least in $N=2$ dimensions (higher dimensions are more complicated, but also illustrated in [19]). $O(N)$-invariant graded [14] Lagrangian spheres correspond to paths in $\mathbb{C}$ between distinct zeros of $p(t)$, missing other zeros, with a continuous choice of lift to $\mathbb{R}$ of the phase $\in \mathbb{R} / 2 \pi \mathbb{Z}$ of the tangent to the path at each point. Special Lagrangians correspond to (spheres fibred over) straight lines, and $O(N)$-invariant hamiltonian isotopies to isotopies of the path in $\mathbb{C}$, with endpoints fixed, not crossing any other zeros of $p$. Extensions correspond to graded Lagrangian connect sums \#, and stability of $L$ to there being no such connect sum $L_{1} \# L_{2}$ hamiltonian isotopic to $L$, with the phase of $L_{1}$ greater than that of $L_{2}$; see Figure 1 , in which $L_{1}$ has phase $\pm \epsilon$ and $L_{2}$ has phase zero.
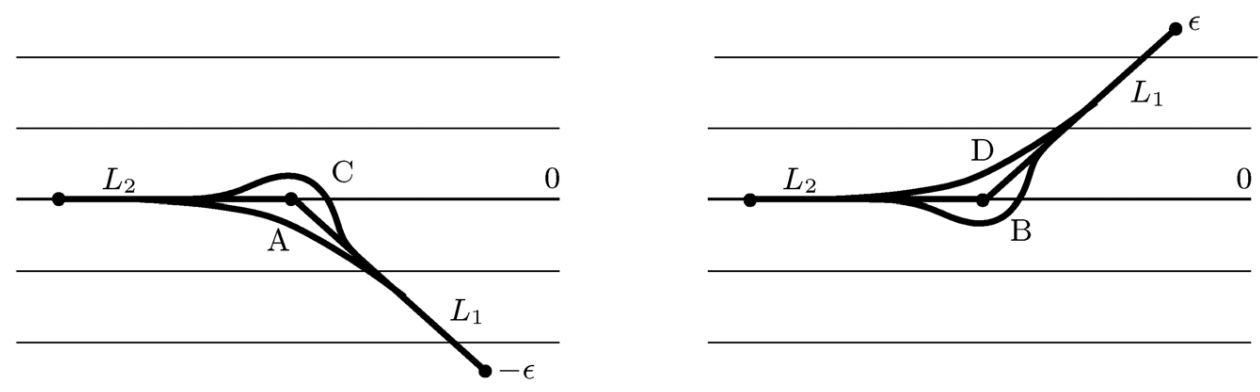

Figure 1: The connect sums $L_{1} \# L_{2}\left(\mathrm{~A}-\right.$ stable, $\mathrm{B}$ - unstable) and $L_{2} \# L_{1}$ (C-unstable, $\mathrm{D}$ - stable) in 2-dimensions, in 2 different complex structures.

Simple examples of loops of complex structures $p$ do indeed exhibit the 
braid monodromy action on the set of such special Lagrangians [19]. For a long time, it was not clear how to extend such naive notions of stability (in terms of injective morphisms or subobjects) to full triangulated categories (where there is no notion of subobject). But the beautiful work of Douglas [6], now axiomatised in [3], purports to give the answer; the purpose of this paper is to show how it works in our $A_{k}$ example, and how it agrees with the naive definitions mentioned above. This also gives a new example where the axioms of [3] hold; in general, examples are hard to find. Known examples work on the B-model side where there is an abelian category (the coherent sheaves), and where the usual notions of (semi)stability of sheaves are well understood and give examples satisfying the axioms (with the semistable objects of the axioms below being the semistable sheaves and their translations) which can be deformed to give more. In the example of this paper, there is no natural abelian category, as we start on the A-model side with the Fukaya category. Using the stable objects of [19], we prove the existence of stability conditions, which in turn shows that the derived Fukaya category is the derived category of many abelian categories.

Our results also apply to the B-model derived category of coherent sheaves, and thus gives a geometric, monodromy background for the mirror braid group action on the derived category described in [15]. The varying complex structure $p$ is then interpreted as a varying complexified Kähler form on the mirror manifold containing an $A_{k}$-chain of spherical objects. In fact, we do not use $p$, but the closely related set of the values it gives to the $A_{k}$-chain of Lagrangians via (2.2):

$$
\left\{\sum_{j=1}^{i} Z\left(L_{j}\right)=\int_{L_{1} \cup \ldots \cup L_{i}} \Omega_{p}\right\}_{i=1}^{k} .
$$

In two dimensions, $Z\left(L_{i}\right)$ is a constant times by the vector in $\mathbb{C}$ between the two zeros of $p$ that form the endpoints of the path over which $L_{i}$ is $S^{1}$-fibred (see (A.1) of [8]). Thus, up to an additive constant in $\mathbb{C}$ which does not affect the homotopy type, the numbers (2.4) and the origin together constitute the distinct roots of $p$ in $\mathbb{C}$, and the map $p \mapsto\left\{0, \sum_{j \leq i} Z\left(L_{j}\right)\right\}$ is a $\mathbb{C}^{*}$ bundle inducing an isomorphism $\pi_{1} / \pi_{1}\left(\mathbb{C}^{*}\right) \rightarrow \pi_{1}\left(C_{k+1}\right) \cong B_{k}$. (In higher dimensions, the relationship is more complicated; for small paths, $Z\left(L_{i}\right)$ is roughly the $(N / 2)$ th power of the vector in $\mathbb{C}$ representing $L_{i}$, which is why it is simpler to stick to two dimensions for our analysis of monodromy.) Instead of the space of $p \mathrm{~s}$, then, we will find the space of stability conditions to be the universal cover of the configuration space $C_{k+1}^{0}$ of $(k+1)$ distinct points of mean zero in $\mathbb{C}$, with fundamental group $B_{k}$. 
Most of the work can be done by just dealing with curves in the plane (with endpoints in the $k+1$ marked points) as in [19], and the stability conditions that emerge axiomatise that of $[18,19]$; i.e. relate to whether or not the path can be pulled "tight" (straight, in the dimension $N=2$ case, or to a special Lagrangian of [16] in general; this was done in [19] by mean curvature flow) without touching one of the other marked points. Unfortunately, one has to deal with slightly more general objects in $D^{b}(F u k(X))$ than can be represented solely by curves in the plane. This is because the curves do not form an abelian, or triangulated, category - in general, one cannot form the kernel or cokernel or cone on a morphism (element of Floer homology) between two Lagrangians. They do form an $A^{\infty}$ category in a complicated way (which involves difficult counting of holomorphic discs with boundaries in the Lagrangians), though an intrinsic formality result [15] means we will not have to worry about the precise $A^{\infty}$ structure. We then have to derive this category, a formal procedure producing cones on abstract morphisms that introduces extra objects not all representable by curves. As proposed in [18], Ext ${ }^{1}$ s are geometrically represented by (graded) connect sums of Lagrangians; what stops us from using this to form geometric representatives of all cones is the fact that some of the $\mathrm{Ext}^{1} \mathrm{~s}$ lead to immersed Lagrangians, whose Floer homology is not well understood, and some lead to different representatives of the same class in the Fukaya category if one either takes the relative connect sum [18] or perturbs and takes a transverse connect sum. It would be nice to find a purely one-dimensional geometric description of all objects of this category.

Nonetheless, our constructions are motivated by pictures for those objects of $D^{b}(F u k)$ that are representable by curves, and so such figures often accompany algebra below without any explanation; these should be helpful to anyone who has read the above background. Unfortunately, such physical arguments do not suffice for the whole category for the $A^{\infty}$ reasons mentioned above, but nonetheless give a good intuitive picture for the axioms [3] that will be familiar to physicists, reminiscent of "marginal stability".

To show that our results are relevant to "real life", we now define our category and show it is indeed faithfully included in the derived Fukaya category of any symplectic manifold with an $A_{k}$-chain of Lagrangian spheres, and in the derived category of any smooth projective variety with an $A_{k^{-}}$ chain of spherical objects [15]. 


\section{The categories.}

We start by defining a simple auxiliary category to define our graded algebra (closely related to the one studied in $[12,10]$ ) whose derived category of dgms will be the triangulated category of study. Compare (2.3).

Definition 3.1. Let $\mathcal{C}=\mathcal{C}_{k}^{N}$ be the $\mathbb{C}$-linear graded category of an $A_{k}$-chain $\left\{E_{j}\right\}_{j=1}^{k}$ in dimension $N$; the category with $k$ spherical objects $E_{j}$,

- $\operatorname{Hom}^{i}\left(E_{j}, E_{j}\right)= \begin{cases}\mathbb{C} & i=0, N \\ 0 & \text { otherwise, }\end{cases}$

and morphisms

- $\operatorname{Hom}^{i}\left(E_{j}, E_{j+1}\right)= \begin{cases}\mathbb{C} & i=1 \\ 0 & \text { otherwise }\end{cases}$

- $\operatorname{Hom}^{i}\left(E_{j+1}, E_{j}\right)= \begin{cases}\mathbb{C} & i=N-1 \\ 0 & \text { otherwise }\end{cases}$

- $\operatorname{Hom}^{i}\left(E_{j}, E_{k}\right)=0,|j-k|>1$.

Denoting by $(j, j \pm 1)$ the generator of $\operatorname{Hom}^{i}\left(E_{j}, E_{j \pm 1}\right)$, we impose the relation that $(j, j \pm 1) \circ(j \pm 1, j)$ is the generator $f_{j}$ of $\operatorname{Hom}^{N}\left(E_{j}, E_{j}\right)$ for all $j$; in particular, then $(j, j+1)(j+1, j)=(j, j-1)(j-1, j)=f_{j}$, and the category has a duality: the pairing $\operatorname{Hom}^{i}(F, E) \otimes \operatorname{Hom}^{N-i}(E, F) \rightarrow \operatorname{Hom}^{N}(E, E) \cong \mathbb{C}$ is perfect for all $E, F \in \mathcal{C}$.

In the usual way, $\mathcal{C}$ defines a graded unital algebra End $\mathcal{C}$ :

$$
A_{k}=A_{k}^{N}=\text { End }\left(\bigoplus_{j} E_{j}\right)=\bigoplus_{i j k} \operatorname{Hom}^{i}\left(E_{j}, E_{k}\right) .
$$

This is a quotient of the path algebra of the quiver in Figure 2. Generators,

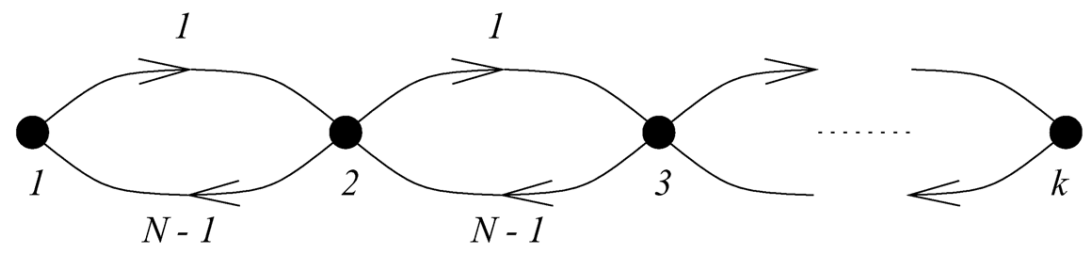

Figure 2.

over $\mathbb{C}$, of the algebra are given by oriented paths, graded as in the Figure, 
with multiplication given by composition of paths (this is the dual picture to the Lagrangian one described in the introduction, with vertices in Figure 2 corresponding to Lagrangians, i.e. paths between zeros of $p$, and arrows corresponding to intersections of Lagrangians, i.e. zeros of $p$ ). The quotient is by the two sided ideal generated by $(i, i-1, i)-(i, i+1, i),(i-1, i, i+1)$ and $(i+1, i, i-1)$ for all $i=2, \ldots, k-1$, in the obvious notation; see [15, Section 4c] for more details. Denote by $e_{i}$ the identity in $\operatorname{Hom}\left(E_{i}, E_{i}\right)$ (i.e. the constant path at the $i$ th node), so that $1=\sum_{i} e_{i}$ and left (right) multiplication by $e_{i}$ is projection onto those paths that begin (end) at the $i$ th node. Similarly, we denote $f_{i}=(i, i-1, i)=(i, i+1, i)$ (or whichever of the two is defined, if $i=1$ or $k$ ).

Now, form the bounded derived category $D\left(A_{k}\right)$ of differential graded (right) modules (dgms) over $A_{k}$; again see [15, Section $\left.4 \mathrm{a}\right]$. This is not the derived category of the abelian category of dgms over $A_{k}$, but the localisation of this category by quasi-isomorphisms (dgm maps that induce isomorphisms on the cohomology graded modules). It is, however, triangulated. Denote by $P_{i}$ the projective (right) module $e_{i} A_{k}$; these form an $A_{k}$-chain in $D\left(A_{k}\right)$ in the sense of Definition 3.1 (with Hom ${ }^{i}$ the $i$ th cohomology of Hom) and so form a subcategory whose cohomology category is isomorphic to the original $\mathcal{C}_{k}$.

Definition 3.2. $D_{k}=D_{k}^{N}$ is the sub-triangulated category of $D\left(A_{k}\right)$ generated by the $P_{i}$; the smallest triangulated subcategory containing the $P_{i}$.

Note that the duality (3.1) induces a "trace map" $\operatorname{Hom}^{N}(E, E) \rightarrow \mathbb{C}$ for all $E \in D_{k}$ such that there exists a perfect pairing $\operatorname{Hom}^{i}(F, E) \otimes$ $\operatorname{Hom}^{N-i}(E, F) \rightarrow \operatorname{Hom}^{N}(E, E) \rightarrow \mathbb{C}$, i.e. a duality

$$
\operatorname{Hom}^{N-i}(E, F)^{*} \cong \operatorname{Hom}^{i}(F, E),
$$

functorial in $E$ and $F$, for all $E, F \in D_{k}$.

This is the category whose stability conditions we will study in this paper. Before we do, we point out that due to the intrinsic formality result of [15], it is contained in the derived Fukaya category of a symplectic manifold containing a (suitably graded) $A_{k}$-chain of Lagrangian spheres, and, mirror to this, in any derived category of coherent sheaves containing a (suitably shifted) $A_{k}$-chain of spherical objects $\left\{E_{i}\right\}$ [15]. Many thanks to Paul Seidel for this argument.

In the first case, the full subcategory $\mathcal{A}$ defined by the $A_{k}$-chain defines a natural dga whose cohomology algebra is our $A_{k}^{N}$. By intrinsic 
formality [15], then, $\mathcal{A}$ is actually quasi-isomorphic to $A_{k}^{N}$. Since the derived Fukaya category contains cones, $\operatorname{Tw}(\mathcal{A})$, the triangulated category of twisted complexes [2] on $\mathcal{A}$, is a full subcategory of the derived Fukaya category. But it is quasi-equivalent to $\operatorname{Tw}\left(A_{k}^{N}\right)$, which is equivalent to the derived category of dgms generated by the projective modules $P_{i}=e_{i} A$ above (since the functor $\operatorname{Hom}\left(\cdot, \oplus_{i} E_{i}\right)$ from $C_{k}^{N}$ to the category of dgms over $A_{k}^{N}=\operatorname{Hom}\left(\oplus_{i} E_{i}, \oplus_{i} E_{i}\right)$ is full and faithful by the Yoneda lemma, and takes the objects $E_{i}$ of $C_{k}^{N}$ to the projectives $P_{i}$ ).

Similarly, given an $A_{k}$-chain in a derived category of coherent sheaves over a smooth projective variety, we can work with the equivalent homotopy category of complexes of quasicoherent injective sheaves with bounded coherent cohomology. This is a dg-category containing cones, so is isomorphic to its own Tw. The $A_{k}$-chain defines a full sub-dg-category defining a dga quasi-isomorphic to its cohomology graded algebra $A_{k}^{N}$ by intrinsic formality. The rest of the argument is then the same.

Proposition 3.3. For $N \geq 2, D_{k}^{N}$ is fully faithfully embedded in the derived Fukaya category of any $2 N$-dimensional symplectic manifold containing an $A_{k}$-chain of spheres; similarly, for the derived category of coherent sheaves on any smooth quasiprojective $N$-dimensional variety with an $A_{k}$-chain of spherical objects.

For instance, in dimension two, we can consider the coherent sheaves over $\mathbb{C}^{2}$ (finitely generated modules over $\mathbb{C}[x, y]$ ) supported at the origin, and the standard $S U(2)$ action of $\mathbb{Z} / k$ on $\mathbb{C}^{2}$. Then, the derived category $D_{0}^{\mathbb{Z} / k}\left(\mathbb{C}^{2}\right)$ of equivariant sheaves supported at the origin is equivalent to the derived category of coherent sheaves on the minimal resolution $\widehat{\mathbb{C}^{2} /(\mathbb{Z} / k)}$ supported on the exceptional set $E[9,4]$. The exceptional locus $E$ is an $A_{k}$-chain of -2spheres whose structure sheaves form an $A_{k}$-chain in the derived category [15], and correspond under the equivalence to the non-trivial irreducible representations of $\mathbb{Z} / k$. So, $D_{k}^{2}$ is embedded in $D_{0}^{\mathbb{Z} / k}\left(\mathbb{C}^{2}\right) \cong D_{E}^{b}\left(\frac{\widehat{\mathbb{C}^{2}}}{\mathbb{Z} / k}\right)$.

\section{Stability.}

In [3], a notion of stability for derived categories is given, axiomatising the proposal of Douglas [6]. Let $K(\mathcal{T})$ denote the Grothendieck group of $\mathcal{T}$.

Definition 4.1. A stability condition $(Z, \mathcal{S S})$ on a triangulated category $\mathcal{T}$ consists of a linear map $Z: K(\mathcal{T}) \rightarrow \mathbb{C}$ and full subcategories $\mathcal{S} \mathcal{S}(\phi) \subset \mathcal{T}$ for each $\phi \in \mathbb{R}$ satisfying the following five axioms: 
(a) for all $\phi \in \mathbb{R}, \mathcal{S S}(\phi+1)=\mathcal{S S}(\phi)[1]$,

(b) if $E \in \mathcal{S S}(\phi)$ then $Z(E)=m(E) \exp (i \pi \phi)$ with $m(E)>0$,

(c) for $0 \neq E \in \mathcal{T}$ there is a finite sequence of real numbers

$$
\phi_{1}>\phi_{2}>\cdots>\phi_{n}
$$

and a collection of triangles

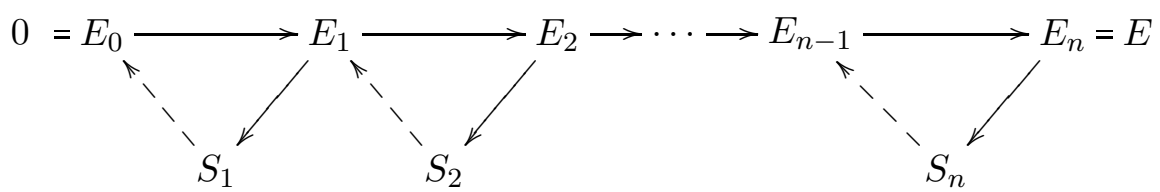

with $S_{i} \in \mathcal{S S}\left(\phi_{i}\right)$ for all $i$,

(d) if $\phi_{1}>\phi_{2}$ and $S_{i} \in \mathcal{S S}\left(\phi_{i}\right)$, then $\operatorname{Hom}_{\mathcal{T}}\left(S_{1}, S_{2}\right)=0$,

(e) the subset

$$
Z\left(\bigcup_{\phi \in \mathbb{R}} \mathcal{S} \mathcal{S}(\phi)\right) \subset \mathbb{C}
$$

has no limit points in $\mathbb{C}$.

The map $Z$ is called the central charge of the stability condition. The objects of the subcategory $\mathcal{S} \mathcal{S}(\phi)$ are said to be semistable of phase $\phi$; the simple semistables are stable, and we denote these by $\mathcal{S}(\phi)$. We call the choice of a lift of the phase $e^{i \pi \phi}$ of such an object $E$ to a real number $\phi$ a grading of $E$. It is an easy exercise to check that the decomposition of a non-zero object $E$ given in (c) is unique; the objects $S_{i}$ are called the semistable factors of $E$. We sometimes call such a collection of triangles a filtration. The mass of $E$ is the positive real number

$$
m(E)=\sum_{i}\left|Z\left(S_{i}\right)\right|
$$

By the triangle inequality, one has $m(E) \geq|Z(E)|$ with equality if $E$ is semistable.

The axioms are modelled on semistability for sheaves on complex curves: on filtering objects by their cohomology sheaves, and these in turn by their Harder-Narasimhan filtrations, we get an example satisfying axiom (c) above.

To make this definition more manageable, we give some conditions that will imply the difficult axiom (c). 
Theorem 4.2. Given $(\mathcal{T}, Z, \mathcal{S}(\phi))$, and defining $\mathcal{S S}(\phi)$ to consist of all possible extensions of elements of $\mathcal{S}(\phi)$, suppose that these satisfy axioms $(a, b, d, e)$ above. Suppose that $\bigcup_{\phi} \mathcal{S}(\phi)=\left\{S_{i}[m]: i=1, \cdots, k, m \in \mathbb{Z}\right\}$, for some finite set of $S_{i}$ which generate $\mathcal{T}$ (i.e. every object in $\mathcal{T}$ is a finite extension of shifts of $S_{i}$ s). Suppose also that for any non-trivial element of $\operatorname{Ext}^{1}(F, E)$ defining a triangle

$$
E \rightarrow C \rightarrow F, \quad \text { with } E \in \mathcal{S}(\phi), F \in \mathcal{S}(\psi), \quad \phi<\psi,
$$

$C$ is either in $\mathcal{S}(\theta)$ with $\theta \in(\phi, \psi)$, or $C=A \oplus B$, with $A \in \mathcal{S}(\alpha), B \in$ $\mathcal{S}(\beta), \phi<\alpha \leq \beta<\psi$. Then $(\mathcal{T}, Z, \mathcal{S S}(\phi))$ satisfy axiom (c), i.e. they define a stability condition.

Remark 4.3. This theorem is surely true more generally: that we get a stability condition if all extensions (4.2) have a Harder-Narasimhan filtration by shifts of $S_{i}$ s (rather than just splitting into two stable objects), but we will only require the above result.

Proof. We have to find a Harder-Narasimhan filtration (c) for any object $E \in \mathcal{T}$. Since the $S_{i}$ generate $\mathcal{T}$, we can find a collection of triangles

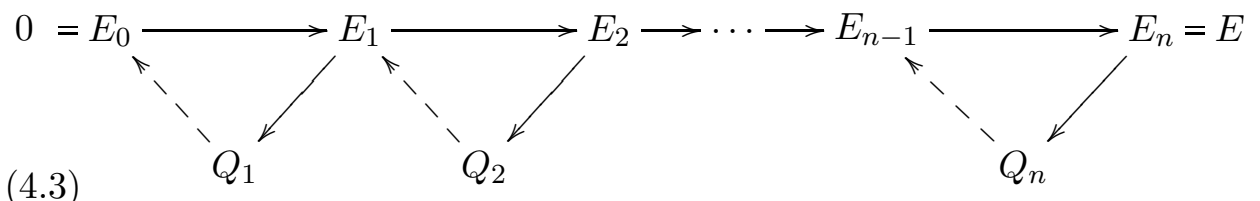

with each $Q_{j} \in \bigcup_{i, m}\left\{S_{i}[m]\right\}$ stable of phase $\phi_{j}$.

Suppose that for some $i, \phi_{i}<\phi_{i+1}$. Replace $E_{i-1} \rightarrow E_{i} \rightarrow E_{i+1}$ in the above filtration by the composition $E_{i-1} \rightarrow E_{i+1}$; this has cone $Q$ fitting into a triangle

$$
Q_{i} \rightarrow Q \rightarrow Q_{i+1}
$$

We can now use the assumption on such extensions, as $\phi_{i}<\phi_{i+1}$ and the $Q_{i}$ are stable.

Either (i) $Q$ is stable, and we replace (4.3) by our new filtration with one less triangle (with $Q_{i}, Q_{i+1}$ replaced by $Q$ of phase $\phi \in\left(\phi_{i}, \phi_{i+1}\right)$ ). We then start the process again, looking for $j$ such that $\phi_{j}<\phi_{j+1}$.

Or (ii) $Q=0$, so $Q_{i+1}=Q_{i}[1]$; in this case, we also remove $E_{i+1}$ from the filtration to give a new filtration with $E_{i-1} \rightarrow E_{i+2}$ in the middle (with cone $Q_{i+2}$ forming the new triangle). 
Or (iii) $Q=Q_{i}^{\prime} \oplus Q_{i+1}^{\prime}$ is the direct sum of two stables of phases $\phi_{i}^{\prime} \geq$ $\phi_{i+1}^{\prime}$ (without loss of generality) in $\left[\phi_{i}, \phi_{i+1}\right]$ (with the closed interval being necessary in case the extension (4.4) is the trivial one). In this case, we define $E_{i}^{\prime}$ by the triangle

$$
E_{i}^{\prime} \rightarrow E_{i+1} \rightarrow Q_{i+1}^{\prime}
$$

where the second arrow is the composition of $E_{i+1} \rightarrow Q$ and $Q=Q_{i}^{\prime} \oplus$ $Q_{i+1}^{\prime} \rightarrow Q_{i+1}^{\prime}$. Then, a small check with the octahedral Lemma gives us a new filtration (4.3) with $E_{i-1} \rightarrow E_{i} \rightarrow E_{i+1}$ replaced by

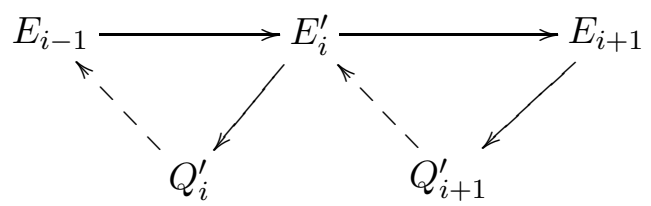

Again, we now start with this filtration.

We claim this procedure terminates; that is after a finite number of steps, we have that $\phi_{i} \geq \phi_{i+1}$ for all $i$. To demonstrate this, we assign to each such filtration a number which is both bounded below and decreases (by some bounded below amount) at each stage. Firstly, we may assume without loss of generality (by replacing $E$ by $E[k]$ for some $k$ if necessary) that each phase $\phi_{i}$ of the $Q_{i}$ in (4.3) is positive.

Then, to (4.3), we can associate the real number $\sum_{k=1}^{n} f(k) \phi_{k}$ for some strictly positive, strictly increasing, concave function $f(x)$. Then, in case (i) above, this clearly decreases, as $\phi<\phi_{i+1}$, so that $f(i) \phi<f(i+1) \phi_{i+1}<$ $f(i) \phi_{i}+f(i+1) \phi_{i+1}$, and the sum over all higher $k \geq i+1$ is also smaller. Case (ii) is even clearer (remembering that all $\phi_{i}>0$ ). Finally, for case (iii), we pick a sufficiently concave function $f$ such that

$$
f(x) \phi+f(x+1) \psi>f(x) \beta+f(x+1) \alpha,
$$

for all $x \geq 0$ and $\phi<\alpha \leq \beta<\psi$ coming from phases of extensions of stable objects as in the assumptions of the Theorem; this is possible since the number of such extensions is finite (up to shifts, which leave the above inequality unaffected).

Then, in case (iii), $f(i) \phi_{i}+f(i+1) \phi_{i+1}>f(i) \phi_{i}^{\prime}+f(i+1) \phi_{i+1}^{\prime}$ (i.e. the above inequality with $\phi_{i}^{\prime}=\beta, \phi_{i+1}^{\prime}=\alpha$ ) ensures that the functional again decreases.

This procedure now terminates as the amount the functional decreases by is bounded below by the discreteness of the phases of the stable objects. 
This gives us a "Jordan-Hölder filtration" of $E$ into stable objects of nonincreasing phases (we use the term as in holomorphic bundle theory or [19] for Lagrangians, rather than the category theoretic terminology). To get the Harder-Narasimhan filtration (c), we bundle together any $Q_{i}$ s of the same phase. That is, if $\phi_{i}=\phi_{i+1}$, replace the $E_{i-1} \rightarrow E_{i} \rightarrow E_{i+1}$ part of the filtration by just $E_{i-1} \rightarrow E_{i+1}$, with one less triangle with cone $Q$ fitting into a triangle

$$
Q_{i} \rightarrow Q \rightarrow Q_{i+1}
$$

By assumption, this means $Q$ is semistable of phase $\phi_{i}=\phi_{i+1}$; now continue the process until the phases are strictly decreasing.

We mention in passing that given a stability condition, we get a family of bounded non-degenerate $t$-structures $\mathcal{F}_{t}=\mathcal{F}_{t+1}[-1]$ on $\mathcal{T}$ given by the full subcategory of objects whose semistable factors (c) all of phase $>t$ [3]. This has as its heart

$$
\mathcal{F}_{t} \cap \mathcal{F}_{t+1}^{\perp}
$$

the full subcategory of objects whose semistable factors (c) all have phase in $(t, t+1]$, so in particular, we can assign a phase $\phi(A) \in(t, t+1]$ to each object $A \neq 0$ of the heart such that $Z(A)=m(A) e^{i \pi \phi(A)}$ with $m(A)>0$. It turns out then [3] that $A$ is semistable if and only if for every subobject $B$ of $A$ in the heart (which is, recall, an abelian category), $\phi(B) \leq \phi(A)$. We may also take $B$ to be stable in this test. We also get (a more standard) Harder-Narasimhan filtration (c) of objects in the heart by semistable objects of the heart.

\section{Our example.}

We first need some technical results about our projective modules $P_{i}$ in $D_{k}^{N}$. Given modules $A, B$ and an element $e$ of $\operatorname{Ext}^{1}(B, A)=\operatorname{Hom}^{0}(B[-1], A)$, we will often denote by $A \# B$ the corresponding extension (i.e. the cone on $B[-1] \rightarrow A$ ) fitting into the triangle

$$
B[-1] \rightarrow A \rightarrow A \# B \rightarrow B
$$

This defines a canonical $\tilde{e} \in \operatorname{Hom}^{0}(A \# B, B)$.

Lemma 5.1. Given $e \in \operatorname{Ext}^{1}(C, A)$ and $f \in \operatorname{Ext}^{1}(C, B)$ defining $A \# C, B \# C$ and $\tilde{e} \in \operatorname{Hom}^{0}(A \# C, C), \tilde{f} \in \operatorname{Hom}^{0}(B \# C, C)$, form $e \cup \tilde{f} \in$ 
$\operatorname{Ext}^{1}(B \# C, A)$ and $f \cup \tilde{e} \in \operatorname{Ext}^{1}(A \# C, B)$. Then the corresponding extensions are isomorphic:

$$
A \#(B \# C) \cong B \#(A \# C) .
$$

Similarly, we have

$$
A \#(B \# C) \cong(A \# B) \# C,
$$

if the classes in $\operatorname{Ext}^{1}(B \# C, A), \operatorname{Ext}^{1}(C, A \# B)$ defining them map to the classes in $\operatorname{Ext}^{1}(B, A), \operatorname{Ext}^{1}(C, B)$ defining $A \# B, B \# C$ respectively.

Proof. The first assertion follows from the diagram of triangles

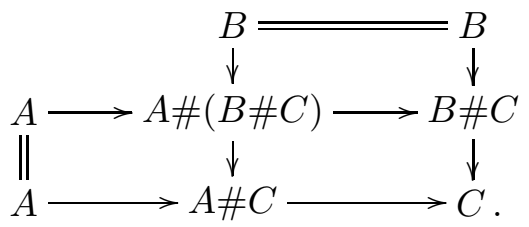

Here, we start with the bottom two rows and left and right-hand columns, and this defines the arrow $A \#(B \# C) \rightarrow A \# C$ by taking cones. The octahedral Lemma then gives the top row, so the central column now shows that $A \#(B \# C) \cong B \#(A \# C)$.

The second statement follows from similar yoga around the diagram

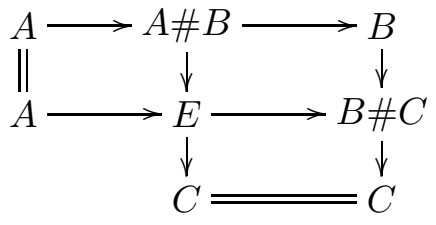

In our use of this Lemma below, \# will be the unique non-trivial extension between the objects concerned. We use the notation $\cong$ for quasiisomorphism and $\mathbb{C}[-n]$ for a copy of $\mathbb{C}$ shifted into degree $n$, so that $\operatorname{Hom}(E, F) \cong \mathbb{C}[-n]$ is equivalent to $\operatorname{Hom}^{*}(E, F)=\mathbb{C}$ for $*=n$ and zero otherwise. The following is best interpreted in terms of pictures such as Figure 3 and the discussion of graded connect sums and relative connect sums in $[18,19]$.

Proposition 5.2. Define $P_{i i}:=P_{i}$, then inductively (on $j \geq i$ ) one can define $P_{i, j+1}:=P_{i j} \# P_{j+1}$ by $\operatorname{Hom}^{1}\left(P_{j+1}, P_{i j}\right)=\mathbb{C}$. 
Moreover, we then have $\operatorname{Hom}\left(P_{k}, P_{i j}\right) \cong\left\{\begin{array}{ll}\mathbb{C}[1-N] & k=i-1 \\ \mathbb{C} & k=i \\ \mathbb{C}[-N] & k=j \\ \mathbb{C}[-1] & k=j+1\end{array}\right.$ and zero otherwise.

Proof. The result is true for $P_{i i}$, i.e. $j=i$, by the fact that the $P_{i}$ form an $A_{k}^{N}$-chain of spherical objects (3.1). Inductively then, assume it is true for $j$, so that we have defined $P_{i, j+1}=P_{i j} \# P_{j+1}$.

Then the triangle

$$
P_{i j} \rightarrow P_{i, j+1} \rightarrow P_{j+1} \rightarrow P_{i j}[1]
$$

gives

$$
\operatorname{Hom}\left(P_{k}, P_{i j}\right) \rightarrow \operatorname{Hom}\left(P_{k}, P_{i, j+1}\right) \rightarrow \operatorname{Hom}\left(P_{k}, P_{j+1}\right) \rightarrow \operatorname{Hom}\left(P_{k}, P_{i j}\right)[1] .
$$

- For $k<i-1, k>j+2$, or $i<k<j$, the second complex is (quasiisomorphic to) zero since all the others are by the induction assumption.

- For $k=i-1$ (respectively $k=i$ ), (5.1) becomes

$$
\mathbb{C}[-d] \rightarrow \operatorname{Hom}\left(P_{k}, P_{i, j+1}\right) \rightarrow 0 \rightarrow \mathbb{C}[1-d],
$$

where $d=N-1$ (respectively $d=0$ ), so that $\operatorname{Hom}\left(P_{k}, P_{i, j+1}\right) \cong \mathbb{C}[-d]$ as required.

- When $k=j$, (5.1) becomes

$$
\mathbb{C}[-N] \rightarrow \operatorname{Hom}\left(P_{j}, P_{i, j+1}\right) \rightarrow \mathbb{C}[1-N] \rightarrow \mathbb{C}[1-N] .
$$

We claim this last map $\operatorname{Hom}^{N-1}\left(P_{j}, P_{j+1}\right) \rightarrow \operatorname{Hom}^{N}\left(P_{j}, P_{i j}\right)$ is an isomorphism, so that $\operatorname{Hom}\left(P_{j}, P_{i, j+1}\right) \cong 0$ as required. This follows from the fact that the composition to $\operatorname{Hom}^{N}\left(P_{j}, P_{j}\right)=\mathbb{C}$, is, by construction, the Yoneda product

$$
\operatorname{Hom}^{N-1}\left(P_{j}, P_{j+1}\right) \otimes \operatorname{Hom}^{1}\left(P_{j+1}, P_{j}\right) \rightarrow \operatorname{Hom}^{N}\left(P_{j}, P_{j}\right),
$$

which is an isomorphism by the duality (3.1).

- For $k=j+1$, we get

$$
\mathbb{C}[-1] \rightarrow \operatorname{Hom}\left(P_{j+1}, P_{i, j+1}\right) \rightarrow \mathbb{C} \oplus \mathbb{C}[-N] \rightarrow \mathbb{C},
$$

with the $\operatorname{Hom}^{0}\left(P_{j+1}, P_{j+1}\right) \rightarrow \operatorname{Hom}^{1}\left(P_{j+1}, P_{i j}\right)$ component of the last map takes the identity to the extension class defining $P_{i, j+1}=P_{i j} \# P_{j+1}$. 
Since this was chosen to be non-trivial, this map is an isomorphism, so $\operatorname{Hom}\left(P_{j+1}, P_{i, j+1}\right) \cong \mathbb{C}[-N]$.

- Finally, when $k=j+2$, we have by $(5.1)$

$$
0 \rightarrow \operatorname{Hom}\left(P_{j+2}, P_{i, j+1}\right) \rightarrow \mathbb{C}[-1] \rightarrow 0
$$

as required. In particular, this now allows us to define $P_{i, j+2}$ and continue the induction.

Thus, $\operatorname{Hom}^{1}\left(P_{i j}, P_{i-1}\right)=\mathbb{C}$ and we can form $P_{i-1} \# P_{i j}$, which by inductive use of Lemma 5.1 is $P_{i-1, j}$. (Alternatively, $\operatorname{Hom}^{0}\left(P_{i-1}, P_{i-1, j}\right)=\mathbb{C}$ with cone $P_{i j}$ gives the same result, as the extension cannot be trivial by the simplicity of $P_{i-1, j}$ demonstrated in Proposition 5.4 below.) More generally, there is a unique extension $P_{i j} \# P_{j k} \cong P_{i k}$ (again see Proposition 5.4, for instance). That is, we may write $P_{i j}=P_{i} \# P_{i+1} \# \ldots \# P_{j}$ without confusion.

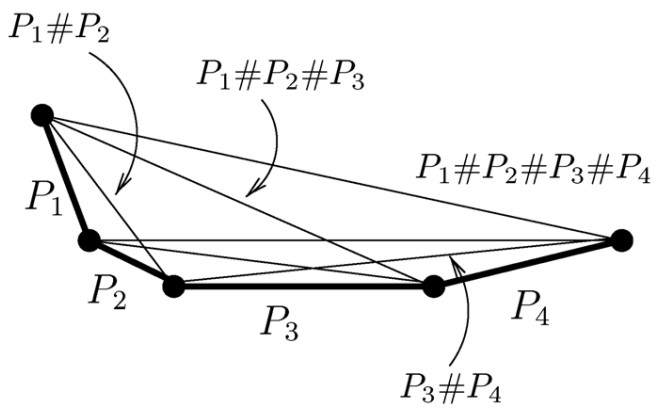

Figure 3: Our collection of stable objects

To define a stability condition on our category $D_{k}^{N}$, we need to define $Z\left(P_{i}\right)$ for all $i$, and the set of (semi)stable objects. We assume that $N \geq 2$, so that there are no non-trivial extensions between any $P_{i}$ and itself. Fix any sequence of positive real numbers $m_{i}$, and a sequence of real numbers

$$
\phi_{1}<\phi_{2}<\ldots<\phi_{k}<\phi_{1}+1 \text {. }
$$

Given any two integers $i \leq j$, we define $P_{i j}:=P_{i} \# P_{i+1} \# \ldots \# P_{j}$ as in the Proposition above, and let $\phi_{i j}$ be the unique real number in the interval $\left[\phi_{i}, \phi_{j}\right] \subseteq\left[\phi_{1}, \phi_{1}+1\right)$ such that $m_{i} e^{i \pi \phi_{i}}+\ldots+m_{j} e^{i \pi \phi_{j}}=m_{i j} e^{i \pi \phi_{i j}}$ for some positive real numbers $m_{i j}$. Note this gives us inequalities

$$
\phi_{i j}<\phi_{k l} \quad \text { if } i<k \text { and } j<l \text {, and } \phi_{i j} \in\left[\phi_{1}, \phi_{1}+1\right) \forall i j \text {. }
$$


Definition 5.3. Fix $N \geq 2$ and $m_{i j}, \phi_{i j}$ as above. Define

$$
Z\left(P_{i}\right):=m_{i} e^{i \pi \phi_{i}}
$$

and extend $Z$ to be defined on all of $K\left(D_{k}\right)=\oplus_{i} \mathbb{Z}_{P_{i}}$ by linearity, so that $Z\left(P_{i j}\right)=Z\left(P_{i}\right)+\ldots+Z\left(P_{j}\right)=m_{i j} e^{i \pi \phi_{i j}}$.

Then define the $P_{i j}[m] \mathrm{s}$ to be the stable objects of phase $\phi_{i j}+m$ in $D_{k}^{N}$, and define the $\mathcal{S S}(\phi)$ s to consist of all direct sums of the stable objects of phase $\phi$.

To show this is a stability condition, we need to understand the Homs between these $P_{i j}$ s, which we do now using Proposition 5.1.

Proposition 5.4. For $i<k<j+1<l+1$, we have

$$
\operatorname{Hom}\left(P_{k l}, P_{i j}\right) \cong \mathbb{C}[-1] \oplus \mathbb{C}[-N]
$$

while if one of the inequalities becomes an equality, we get only one of the two summands: for $i=k<j+1<l+1$ and $i<k<j+1=l+1$, we get $\mathbb{C}[-N]$, while for $i<k=j+1<l+1$ we get $\mathbb{C}[-1]$.

The duality $\operatorname{Hom}\left(P_{k l}, P_{i j}\right) \cong \operatorname{Hom}\left(P_{i j}, P_{k l}\right)^{\vee}[-N]$ (3.1) applied to (5.4) determines more Homs. All others are zero apart from $\operatorname{Hom}\left(P_{i j}, P_{i j}\right) \cong$ $\mathbb{C} \oplus \mathbb{C}[-N]$ : the $P_{i j}$ are spherical.

Proof. Building up $P_{i j}=P_{i, j-1} \# P_{j}=P_{i} \# P_{i+1, j}$ inductively and using Proposition 5.1 gives this result very easily. We give the example of most interest to us: $i<k=j+1<l+1$.

Using $\operatorname{Hom}\left(P_{r}, P_{i j}\right) \cong 0$ for $r>j+1$ (5.1), it is easy to show inductively that $\operatorname{Hom}\left(P_{r l}, P_{i j}\right)=0$ for $r>j+1$ (where if $l<r$, we define $P_{r l}:=0$ ). Then applying $\operatorname{Hom}\left(\cdot, P_{i j}\right)$ to

$$
P_{j+1} \rightarrow P_{j+1, l} \rightarrow P_{j+2, l}
$$

gives $(5.1)$

$$
\mathbb{C}[-1] \rightarrow \operatorname{Hom}\left(P_{j+1, l}, P_{i j}\right) \rightarrow 0 \rightarrow \mathbb{C} .
$$

Thus, $\operatorname{Hom}\left(P_{j+1, l}, P_{i j}\right)=\mathbb{C}[-1]$ as required.

The fact that the $P_{i j}$ are spherical is also proved inductively from the observation that if $A$ and $B$ are spherical with $\operatorname{Hom}(B, A)=\mathbb{C}[-1]$, then 
the corresponding extension $A \# B$ is also spherical (the connect sum of two spheres is a sphere!): from the triangle $A \rightarrow A \# B \rightarrow B$, we get

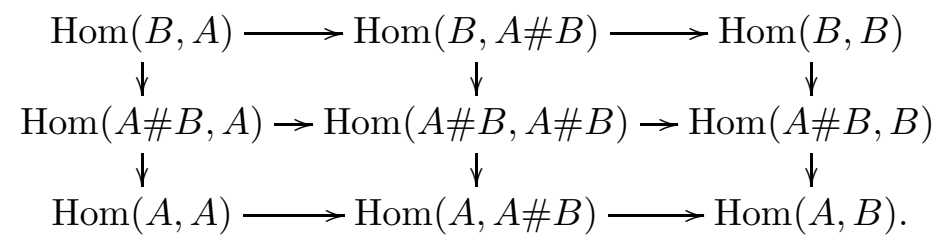

In the first column, the connecting map $\operatorname{Hom}^{0}(A, A) \rightarrow \operatorname{Hom}^{1}(B, A)$ takes the identity to the generator, by definition of the non-trivial extension. Since $A$ is spherical, this makes $\operatorname{Hom}(A \# B, A) \cong \mathbb{C}[-N]$. Similarly, with the last column, using the functorial duality $\operatorname{Hom}(E, F)^{\vee} \cong \operatorname{Hom}(F, E)[N]$. So the central row becomes

$$
\mathbb{C}[-N] \rightarrow \operatorname{Hom}(A \# B, A \# B) \rightarrow \mathbb{C},
$$

from which it follows that $A \# B$ is spherical, since $N \geq 2$.

Finally, we can prove that we are in the situation of Theorem 4.2.

Theorem 5.5. Definition 5.3 defines a stability condition (4.1) on $D_{k}^{N}$.

Proof. Axioms (a), (b) and (e) of (4.1) are immediate from Definition 5.3, while (d) follows from Proposition 5.4 and the inequalities (5.3). Axiom (c) will follow from Theorem 4.2 if we can show that any non-trivial extension $E \rightarrow C \rightarrow F$, with $E, F$ stable of phases $\phi<\psi, C$ is either stable (of phase $\theta \in(\phi, \psi))$, or a sum of stables $C=A \oplus B$, with $A \in \mathcal{S}(\alpha), B \in \mathcal{S}(\beta), \phi<$ $\alpha \leq \beta<\psi$.

Such extensions are given by the shifts of the Homs computed in Proposition 5.4. Only the Homs listed there in degrees 0 and 1 interest us: if there is a non-zero $\operatorname{Hom}^{n}(F, E)$ with $n \geq 2$ and the phases of $E$ and $F$ less than 1 apart (5.3) then this gives rise to an extension in $\operatorname{Hom}^{1}(F, E[n-1])$ with the phase of $E[n-1]$ greater than the phase of $F$, which therefore does not concern us. Note, however, that by duality, Homs of degree $N, N-1$ give rise to Homs of degree 0,1 , in the opposite direction, that we do need to consider. This gives us 5 cases to check (there are also selfHoms to consider, but these just give $P \# P[1] \cong 0$ ).

- $P_{i j} \# P_{j+1, l}$, and their shifts. We have already observed that this gives the single stable object $P_{i l}$ with phase $\phi_{i l} \in\left(\phi_{i j}, \phi_{j+1, l}\right)$. 
- $P_{k l} \#\left(P_{k j}[1]\right), j<l$, and their shifts. This extension comes from the element of $\operatorname{Hom}^{0}\left(P_{k j}, P_{k l}\right)$ in the triangle $P_{k j} \rightarrow P_{k l} \rightarrow P_{j+1, l}$, and so is isomorphic to $P_{j+1, l}$, with phase $\phi_{j+1, l} \in\left(\phi_{k l}, \phi_{k j}+1\right)$.

- $P_{k l} \#\left(P_{i l}[1]\right), i<k$, and their shifts. This extension comes from the element of $\operatorname{Hom}^{0}\left(P_{i l}, P_{k l}\right)$ in the triangle $P_{i, k-1} \rightarrow P_{i l} \rightarrow P_{k l}$, and so is isomorphic to $P_{i, k-1}[1]$, with phase $\phi_{i, k-1}+1 \in\left(\phi_{k l}, \phi_{i l}+1\right)$.

- $P_{i j} \# P_{k l}, i<k<j+1<l+1$, and their shifts. This element of Hom ${ }^{1}$ is in the image of $\operatorname{Hom}^{1}\left(P_{k l}, P_{i, k-1}\right)$ (that defines $\left.P_{i, k-1} \# P_{k l}=P_{i l}\right)$ via the map $P_{i, k-1} \rightarrow P_{i j}$ (with cone $P_{k j}$ ). So, we induce a diagram

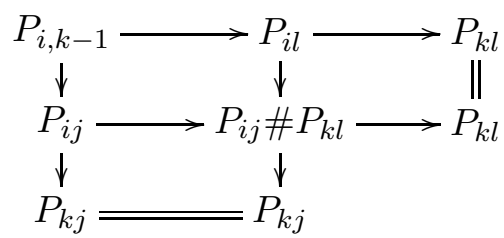

where the bottom row is induced from the octahedral Lemma. So the central column shows that $P_{i j} \# P_{k l} \cong P_{k j} \oplus P_{i l}$, as there are no non-trivial extensions between these two objects, by Proposition (5.4). (See also Figure 4.) The inequalities (5.3) give the required $\phi_{k j}, \phi_{i l} \in\left(\phi_{i j}, \phi_{k l}\right)$.

- $P_{k l} \#\left(P_{i j}[1]\right), i<k<j+1<l+1$, and their shifts. Similarly, the diagram

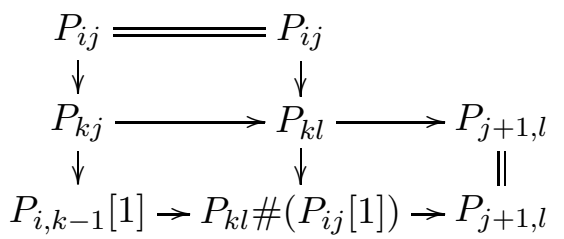

of the standard morphisms we have already seen shows that $P_{k l} \# P_{i j}[1] \cong$ $P_{j+1, l} \oplus P_{i, k-1}[1]$ since there are no extensions between these two objects. Again (5.3) shows that $\phi_{j+1, l}, \phi_{i, k-1}+1 \in\left(\phi_{k l}, \phi_{i j}+1\right)$.

\section{Deforming the stability condition.}

In this section, we determine an entire connected component of the space of stability conditions in dimension $N=2$ and connect it with braid groups of autoequivalences $[12,10,15]$. The proofs are a little brief; in particular, they make use of the description of stability conditions mentioned in (4.5) and proved in [3]. 

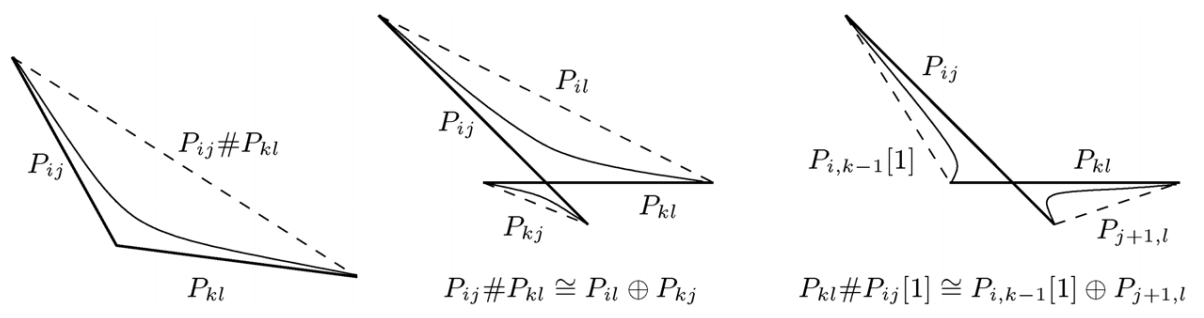

Figure 4: Connect sums of stable objects $P_{i j}$ and $P_{k l}$ of increasing phase are (direct sums of) stable objects: they (the curved lines) can be deformed to the dashed straight lines.

In [3], the space of stability conditions is shown to be a metric space in a natural way, such that the mass function (4.1) is continuous. Using the description of stability described in the paragraph (4.5), the space is shown locally, about any given stability condition, to be isomorphic to the space $\operatorname{Hom}(K(\mathcal{T}), \mathbb{C})$ of $Z$ s. In fact, it is shown to be a cover of the space of $Z \mathrm{~s}$ minus those where a mass (4.1) goes to zero. That is, the stability condition deforms even through walls of $Z \mathrm{~s}$ where some objects become unstable and others stable, so long as $Z$ of a stable object does not become zero. We will show that, in our case, this means that the points 0 and $Z\left(P_{1 j}\right)=\sum_{i=1}^{j} Z\left(P_{i}\right)$ are distinct, i.e., geometrically, the "endpoints" of our special Lagrangians are distinct - no stable object's mass has gone to zero. On looping round such a zero (a generator of the braid group) back to the same $Z$, we will find that the stability condition has changed, as the set of stable objects undergoes a "Dehn twist" [15], at least in the easiest case to analyse, dimension $N=2$, to which we will restrict from now on.

To do this, we will need show that under deformations of $Z$, our set of stable objects keep the following properties of our initial set of stable objects (5.3).

Definition 6.1. The set of stable objects of a stability condition on $D_{k}^{2}$ is called simple if it is the set of shifts of $k(k+1) / 2$ distinct spherical objects $Q_{i j}, 1 \leq i \leq j \leq k$, satisfying the following conditions. $\left[Q_{i j}\right]=\left[P_{i j}\right]$ in K-theory, there is a single Hom in some degree between $Q_{a b}$ and $Q_{b+1, c}$, a single Hom in some degree between $Q_{a b}$ and $Q_{a c}(c \neq a)$, and the Euler characteristic

$$
\chi\left(Q_{a b}, Q_{c d}\right):=\sum_{i}(-1)^{i} \operatorname{dim} \operatorname{Hom}^{i}\left(Q_{a b}, Q_{c d}\right)=0 \quad \text { for } \quad a<c \neq b+1 .
$$


We call the stability condition simple if its set of stable objects is simple and its semistable objects are direct sums of stable objects of the same phase.

We want to show next that the stability condition remains simple on passing through walls of semistability.

We say that $Z \in \operatorname{Hom}\left(K\left(D_{k}\right), \mathbb{C}\right)$ lies on a codimension one wall if $Z\left(\left[P_{i j}\right]\right) \neq 0 \forall i, j$, at least 2 of the classes $\left[P_{i j}\right]$ have the same phase $(\bmod 1)$, and at most 3 , in which case, the three must be linearly dependent (and so of the form $\left[P_{a b}\right],\left[P_{b+1 c}\right],\left[P_{a c}\right]$ - notice that for the sum of two $\left[P_{i j}\right]$ classes to equal the class of another, the two classes must be of the form $\left[P_{a b}\right],\left[P_{b+1 c}\right]$.)

We can talk locally about "sides" of such a wall depending on the sign of the difference in sign of these two phases.

Proposition 6.2. If $P, Q$ are distinct stable objects in a simple stability condition, close to, and on one side of, a codimension one wall, and whose phases coincide on the wall, then the total dimension of $\operatorname{Hom}^{*}(P, Q)$ is at most 1.

If the total dimension of $\operatorname{Hom}^{*}(P, Q)$ is exactly 1, then there is a further stable object $R$ of the same phase on the wall, and, on reordering $P, Q, R$ if necessary, the Hom is of degree 1 and $R \cong P \# Q$.

Proof. We need to rule out there being two or more Homs between $P$ and $Q$. In this case, by the definition of codimension one wall and simple (6.1), there are no other K-theory classes of stable objects whose phases tend to those of $[P],[Q]$ on the wall.

Shifting $Q$ and swapping $P, Q$ if necessary, and moving closer to the wall, we can assume that the phase of $Q$ is more than that of $P$, and that there are no stable objects of phase in between. Thus, by stability and Serre duality, $\operatorname{Hom}^{i}(Q, P)=0$ for $i \leq 0$ and $i \geq 3$. By the vanishing of the Euler characteristic (6.1), then, there must be equal numbers of Homs in degrees 1 and 2; pick a non-zero element of $\operatorname{Hom}^{1}(Q, P)$ and form the extension $P \# Q$. Using the description of stability given in the paragraph (4.5), if $P \# Q$ were unstable, there would be a stable object, with a non-zero Hom to $P \# Q$, of phase between those of $P, Q$. But this is a contradiction, so that $P \# Q$ is stable - another contradiction, since its K-theory class is $[P]+[Q]$, which does not contain a stable object.

So we need only consider the case where there is a single Hom from $Q$ to $P$, which by axiom (d) and Serre duality must be in degree 1 or 2 (for the phase of $Q$ greater than that of $P$ ). There are now 3 stable objects $P, Q, R$ whose phase is tending to the same value on the wall. We work with the 
semistability criterion in the abelian category (4.5) (for a suitable value of $t$ so that it contains $P, Q, R)$. Without loss of generality, we will assume that the masses (4.1) of $P, Q$ are less than or equal to that of $R$ on the wall.

There can be no element of $\operatorname{Hom}^{2}(Q, P) \cong \operatorname{Hom}^{0}(P, Q)^{*}$, as the image in $Q$ of any Hom from $P$ (i.e. the cokernel of its kernel in the abelian heart) would have a filtration by stable objects of phase between those of $P$ and $Q$, and of strictly smaller mass.

So $\operatorname{Hom}^{1}(Q, P)=\mathbb{C}$, and we can form $P \# Q$. Its Harder-Narasimhan filtration in the abelian category (4.5) is of semistable objects of phase between those of $P$ and $Q$; i.e. of direct sums of shifts of $P, Q, R$. Since the extension is non-trivial and the masses of $P, Q$ are less than or equal to that of $R$, it follows that the filtration is just $R$, that is $P \# Q=R$. We claim that there is a single Hom between $R$ and either of $P$ and $Q$; for instance, $\operatorname{Hom}(R, P)$ fits into long exact sequence

$$
\operatorname{Hom}^{i}(Q, P) \rightarrow \operatorname{Hom}^{i}(P \# Q, P) \rightarrow \operatorname{Hom}^{i}(P, P) \rightarrow \operatorname{Hom}^{i}(Q, P) \rightarrow \ldots,
$$

in which the identity in $\operatorname{Hom}(P, P)$ maps to the generator of $\operatorname{Hom}^{1}(Q, P)$. Thus, $\operatorname{Hom}(P \# Q, P) \cong \mathbb{C}[2]$. Similarly, $\operatorname{Hom}(Q, P \# Q) \cong \mathbb{C}[2]$.

Proposition 6.3. On crossing a codimension one wall, a simple stability condition remains simple.

Proof. Suppose the phases of two stable objects $P$ and $Q$ (on one side of the wall, where the phase of $P$ is less than that of $Q$, without loss of generality) coincide on the wall. If there are no Homs between them, it is easy to see that there are no other such stable objects, and the set of (semi)stable objects does not change across the wall; in particular, $P$ and $Q$ remain stable (see, for example, [3] for more details, or use the equivalent definition of stability in the paragraph (4.5)).

So by Proposition 6.2, we need only consider the case where there is a single Hom from $Q$ to $P$, of degree one, and $R=P \# Q$ is also stable.

Then for $P, Q$ to become unstable as we cross the wall, they must have a filtration by stable objects of strictly smaller mass and the same phase on the wall. But because this is a codimension one wall and $R$ has greater mass, no such objects exist.

$R$ becomes unstable on the other side of the wall. But there we can use Serre duality to form a unique non-trivial extension $Q \# P$, which is similarly stable on that side of the wall as in the proof of Proposition 6.2. $Q \# P$ is also spherical as in the proof of Proposition 5.4. Since no other stable objects are 
affected, we claim that this new set of stable objects (with each shift $P \# Q[r]$ replaced by $Q \# P[r])$ is also simple. The K-theory class of $Q \# P$ is the same as that of $P \# Q$, and its Homs to $P$ and $Q$ are one dimensional as in the last paragraph of the proof of Proposition 6.2. To satisfy Definition 6.1, then, we must finally check that for any stable object $E$ with $\chi(E, P \# Q)=0(6.1)$, we also have $\chi(E, Q \# P)=0$. But by the exact sequence $\operatorname{Hom}^{i}(E, Q) \rightarrow$ $\operatorname{Hom}^{i}(E, Q \# P) \rightarrow \operatorname{Hom}^{i}(E, P) \rightarrow \operatorname{Hom}^{i+1}(E, Q) \rightarrow \ldots$, we see that

$$
\chi(E, Q \# P)=\chi(E, Q)+\chi(E, P)=\chi(E, P \# Q)=0,
$$

as required.

While we have analysed crossing only codimension one walls, the fact from [3], mentioned above, that locally the space of stability conditions is isomorphic to the space of $Z \mathrm{~s}$ means that there is no monodromy around codimension 2 walls, and whenever $Z$ does not lie on a wall, the stability condition is simple. In particular, then, there is always a stable object in the K-theory class $\left[P_{i j}\right]$ away from the finite number of walls. Combined with the results on deforming stability conditions [3], which can always be done until a stable object's mass goes to zero, we find the connected component of our stability conditions (5.3) is a cover of the space of $Z$ s such that $Z\left(\left[P_{i j}\right]\right) \neq 0$ for all $i \leq j$. Plotting the points 0 and $Z\left(\left[P_{1 i}\right]\right)$ for all $i$, and translating them to have mean zero, this space in turn covers the configuration space $C_{k+1}^{0}$ of $(k+1)$ distinct points in $\mathbb{C}$ with centre of mass the origin. We now prove slightly more.

Theorem 6.4. Via the above map, the connected component of the stability conditions (5.3) is the universal cover of the configuration space $C_{k+1}^{0}$. The deck transformations are given by the $B_{k}$ action of [10, 15].

Proof. We want to check that the result of going round loops in configuration space is the braid group action of $[10,15]$. It is sufficient to check this for a suitable choice of generators; namely, we pick a stability condition as in (5.3) in which the mass $\left|Z\left(P_{i i}\right)\right|$ of the stable object $P_{i}$ is strictly smaller than that of all other stable objects, and we move $Z$ by rotating $Z\left(P_{i}\right)$ anticlockwise through $\pi$ radians while fixing $Z$ of stable objects of different endpoints. As we rotate $Z\left(P_{i}\right)$, the phases of the stable objects $P$ with an endpoint $i-1$ or $i$ will also change (by no more than $\epsilon=\sin ^{-1}\left(\left|Z\left(P_{i}\right)\right| /|Z(P)|\right)$, by the triangle inequality); we also assume that $\left|Z\left(P_{i i}\right)\right|$ is so small that no two phases of classes $\left[P_{k l}\right] \neq\left[P_{i}\right]$ coincide under this rotation; i.e. we take all 
the phases of the classes $\left[P_{k l}\right]$ to be distinct, and $\epsilon$ to be smaller than the smallest difference in such phases.

Note that for this choice of stability condition and stable object $P_{i}$, there are no other stable objects with 2 Homs to $P_{i}(5.1)$; they either have no Homs or one. As we rotate $Z\left(P_{i}\right)$ and follow the stability condition below, we can see that this property is preserved, as by design no stable objects' phases become equal, except to the phase of $P_{i}$, so no stable objects change except via their interaction with $P_{i}$ described now.

As we rotate $Z\left(P_{i}\right)$ and cross walls (of codimension one only, without loss of generality, by perturbing the loop if necessary), stable objects with no Homs are unaffected, while those with one Hom are altered as in the proof of the last Proposition.

That is, as the phase of $Z\left(P_{i}\right)$ reaches that of some other pair of stable objects $E, F$ ( $F$ of smaller mass) with $\operatorname{Hom}\left(P_{i}, E\right) \cong \mathbb{C}[0], \operatorname{Hom}\left(P_{i}, F\right) \cong$ $\mathbb{C}[-1]$ and $E \cong P_{i} \# F$. On passing through the wall, the stable object $E$ is replaced by $F \# P_{i}$, and all other stable objects are left unchanged.

But we claim that

$$
T_{P_{i}}\left(P_{i} \# F\right) \cong F \quad \text { and } \quad T_{P_{i}} F \cong F \# P_{i},
$$

where $T_{P_{i}}$ is the Dehn twist about the spherical object $P_{i}$; an equivalence of triangulated categories [15]. $T_{P_{i}}(E)$ sits in the triangle

$$
P_{i} \otimes \operatorname{Hom}\left(P_{i}, E\right) \rightarrow E \rightarrow T_{P_{i}}(E)
$$

where the first map is evaluation. Since $\operatorname{Hom}\left(P_{i}, P_{i} \# F\right) \cong \mathbb{C}[0]$ and $\operatorname{Hom}\left(P_{i}, F\right) \cong \mathbb{C}[-1]$, applying this to $E=P_{i} \# F$ and $E=F$ gives the standard triangles

$$
P_{i} \rightarrow P_{i} \# F \rightarrow F \quad \text { and } \quad P_{i}[-1] \rightarrow F \rightarrow F \# P_{i}
$$

respectively, proving the claim.

So on listing the stable objects in ascending phase, we find that the subsequence $P_{i}, E, F$ is replaced by $F, F \# P_{i}, P_{i}$, i.e. by $T_{P_{i}} E, T_{P_{i}} F, P_{i}$.

The same is true when the phase of $P_{i}$ passes through that of the other stable objects $P$ with no Homs to $P_{i}$ : they are left unaffected, just as under $T_{P_{i}}: T_{P_{i}} P \cong P$.

As we rotate $Z\left(P_{i}\right)$ through $\pi$, its phase crosses the phase of all stable objects (after a suitable shift), and so, we end up with the list of stable objects of ascending phase $P_{i}, A, B, \ldots$ being replaced by $T_{P_{i}} A, T_{P_{i}} B, \ldots, P_{i}$. Equivalently, as $P_{i}$ s phase has increased by 1 , we have $P_{i}[-1], T_{P_{i}} A, T_{P_{i}} B, \ldots$ But 
$T_{P_{i}} P_{i} \cong P_{i}[-1]$, so we have altered the set of stable objects (and $Z$ ) by the action of $T_{P_{i}}$, as claimed.

Finally, since the $B_{k}$ action of $[10,15]$ is faithful not just on the triangulated categories, but also on $A_{k}$-chains such as the stable objects $\left\{P_{i}\right\}[15$, Theorem 4.13], we see that the cover of $C_{k+1}$ that we get is the universal cover.

We end by noting that if we take an element of the braid group which acts trivially on K-theory (and so on $Z$ ), then the two stability conditions differ only by their set of stable objects. In our case, these stable objects differed by an autoequivalence of the triangulated category. More generally, if the axiomatic notion of stability of [3] is to agree with the physical notion of stability (and the geometric conjectures in [18]), this would have to hold more generally; that is, one might conjecture that two stability conditions with the same central charge on a "Calabi-Yau category" (one with a "trace map" $\operatorname{Hom}^{N}(E, E) \rightarrow \mathbb{C}$ inducing a functorial duality $\operatorname{Hom}(A, B)^{*} \cong \operatorname{Hom}(B, A)[N]$ for all $\left.A, B\right)$ should differ by an autoequivalence of the category that is the identity on its numerical K-theory.

\section{Acknowledgements.}

This project started out as joint work with Tom Bridgeland, who pulled out due to laziness and my misuse of the term "Jordan-Hölder filtration". I owe him a great deal for discussions, an advanced copy of his axioms [3], and the many errors that he spotted. I would also like to thank Ludmil Katzarkov and Paul Seidel for extremely useful conversations. The author is supported by a Royal Society university research fellowship.

\section{References.}

[1] P. S. Aspinwall and M. R. Douglas, D-Brane Stability and Monodromy, J. High Energy Phys. 5 (2002), 31. hep-th/0110071.

[2] A. Bondal and M. Kapranov, Framed triangulated categories, Math. USSR-Sb. 70 (1991), 93-107.

[3] T. Bridgeland, Stability conditions on triangulated categories, preprint math.AG/0212237. 
[4] T. Bridgeland, A. King and M. Reid, Mukai implies McKay: the McKay correspondence as an equivalence of derived categories, J. Amer. Math. Soc. 14 (2001), 535-554. math.AG/9908027.

[5] M. R. Douglas, D-branes, categories and $N=1$ supersymmetry. Strings, branes, and M-theory. J. Math. Phys. 42 (2001), 2818-2843. hepth/0011017.

[6] M. R. Douglas, Dirichlet branes, homological mirror symmetry, and stability, Proceedings of the International Congress of Mathematicians, Vol. III (Beijing, 2002), 395-408, Higher Ed. Press, Beijing, 2002. math.AG/0207021.

[7] P. Griffiths and J. Harris, Principles of algebraic geometry. Wiley, New York. 1978.

[8] S. Hosono, Central charges, symplectic forms, and hypergeometric series in local mirror symmetry, preprint hep-th/0404043.

[9] M. Kapranov and E. Vasserot, Kleinian singularities, derived categories and Hall algebras, Math. Ann. 316 (2000), 565-576. math.AG/9812016.

[10] M. Khovanov and P. Seidel, Quivers, Floer cohomology, and braid group actions, J. Amer. Math. Soc. 15 (2002), 203-271. math.QA/0006056.

[11] M. Kontsevich, Homological Algebra of Mirror Symmetry, International Congress of Mathematicians, Zürich 1994. Birkhäuser, 1995. alggeom/9411018.

[12] R. Rouquier and A. Zimmermann, Picard groups for derived module categories, Proc. London Math. Soc. 87 (2003), 197-225.

[13] P. Seidel, Lagrangian two-spheres can be symplectically knotted, J. Differential Geom. 52 (1999), 145-171. math.DG/9803083.

[14] P. Seidel, Graded Lagrangian submanifolds, Bull. Soc. Math. France. 128 (2000), 103-146. math.SG/9903049.

[15] P. Seidel and R. P. Thomas, Braid group actions on derived categories of sheaves, Duke Math. J. 108 (2001), 37-108. math.AG/0001043.

[16] A. Shapere and C. Vafa, BPS structure of Argyres-Douglas superconformal theories, preprint hep-th/9910182. 
[17] B. Szendröi, Artin group actions on derived categories of threefolds, J. Reine Angew. Math. 572 (2004), 139-166. math.AG/0210121.

[18] R. P. Thomas, Moment maps, monodromy and mirror manifolds, Symplectic geometry and mirror symmetry (Seoul, 2000), 467-498, World Sci. Publishing, 2001. math.DG/0104196.

[19] R. P. Thomas and S.-T. Yau, Special Lagrangians, stable bundles and mean curvature flow, Communications in Analysis and Geometry 10, 1075-1113, 2002. math.DG/0104197.

Department of Mathematics

IMPERIAL COLLEGE

180 QueEn's Gate

LONDON SW7 2BZ. UK.

E-mail address: rpwt@ic.ac.uk 